\title{
Designing a useful and usable mobile EMR application through a participatory design methodology: a case study
}

\author{
Robin De Croon, Joris Klerkx, Erik Duval \\ Department of Computer Science, KU Leuven \\ Celestijnenlaan 200A \\ BE-3000 Leuven \\ Email: \{robin.decroon, joris.klerkx, erik.duval\}@cs.kuleuven.be
}

\begin{abstract}
An electronic medical record (EMR) supports the general practitioner (GP) in decision-making. In this paper, we report on our study, in which we analyze and identify user requirements for a mobile EMR application. By relying on a participatory design approach [1], we were able to identify the most relevant patient data for house visits by a GP. Our findings further refine earlier results from similar research [2], [3] by narrowing down the most important information for GPs that should be visible immediately. We also identify five core considerations for the use of a mobile EMR application during a house visit. These five considerations are translated into five requirements: exploiting mobile affordances, immediate availability, quick overview of most relevant data, easy entry of new patient data and the importance of e-prescriptions. We iteratively designed the user interface for a mobile application that meets these requirements. Thus, by taking advantage of the affordances of a mobile device, including context sensing, we optimized the way a GP can use a mobile EMR application.
\end{abstract}

\section{INTRODUCTION AND MOTIVATION}

An electronic medical record (EMR) can facilitate the general practitioner (GP) in decision making. However, through structured interviews, our study shows that several problems such as battery life, internet connection, outdated laws and non-optimized user interfaces, inhibit Belgian GPs use an EMR during house visits. A patient contact is, therefore, rarely registered and error prone, which is an important problem. Much information (e.g. medication) is not registered in the patient's file, resulting in incomplete profiles. Barach et al. [4] report that nearly 100,000 individuals per year in the US die of preventable medical errors or 'adverse drug events'. To counter such events, the British Department of Health recommends a wider use of electronic tools [5]. Other benefits of an EMR include effective communication towards the patient [6] and sharing of patient information among various caregivers. It is therefore clear that more research is necessary to ensure that GPs can use EMRs in all situations, both in their practice as during house visits.

This paper shows how we used a participatory design methodology, as described by Spinuzzi [1], to determine considerations, requirements and solutions of a mobile health application that will enable GPs to effectively use a mobile EMR in Belgium. This design methodology provides user feedback from the initial phase onwards and also allows to gather several facts about design situations we may otherwise not been aware of; e.g. we noticed a major difference between the different GP generations (Section VIII). In line with this methodology, our work consisted of three stages to design a usable and useful application [1]: 1) initial exploration of work, 2) discovery processes and 3) prototyping. In the first stage, we have familiarized ourselves with existing mobile and desktop/laptop EMRs. Contrary to latter, mobile devices offers several mobile affordances such as multi-touch, GPS, longer battery life, lower weight, smaller size, etc. The discovery process is conducted in collaboration with two GPs and a company that develops medical software. We validated our prototypes in three steps: (i) an initial design was evaluated with nineteen GPs, (ii) a second evaluation focused primarily on usability and was therefore conducted with six usability experts and finally, (iii) we worked together with 53 last year general practice students to evaluate the final outcome.

The remainder of this paper starts with a review of related work. First, we present our contributions. Next, the methodology is explained in Section IV. Then, the participatory design process is explained in Section $\mathrm{V}$ and early prototypes are presented in Section VI. Thereafter, the usability evaluation is explained in Section VII. The results are discussed in Section VIII. Finally, we conclude with lessons learned and offer some suggestions for future work.

\section{RELATED WORK}

\section{A. GPs' needs}

There have been various efforts to help GPs with technology. The work from Abd Ghani et al. [2] identifies requirements for such applications. Besides developing an architectural framework for an electronic health record (EHR), they also documented user needs. Based on interviews with 30 GPs from different health clinics and polyclinics, they identified the most important patient data during a doctor-patient consultation. Similar to our research, they also reviewed some existing clinical information systems. GPs ranked diagnosis and problems, allergies information, lab test result, radiology report, medication information, complaints information, symptoms, vital signs, family history, social history, date of previous visit and onset date of diagnosis as very important. Since we developed an interface for a mobile EMR application with a smaller screen, we refined these results to a smaller subset as described in Section VI. Because each country has its own 
regulations and cultural differences, user needs could differ. We compared the study from Abd Ghani et al. [2] with a more local study [3] on the same topic. Some patients are always treated on a house visit and for these patients, GPs do not register information in their EMR. Plancke [3] determined the patient information that is needed on a patient file that is stored at the home of the patient himself. We want to know if this information is also needed for a mobile EMR application that can be taken on a house visit. Plancke [3] interviewed 50 Belgian GPs and came to a similar result: active problems, active diseases, active medication, allergies, intolerances, medical prehistory, therapeutic history and recent lab results are the most needed information in a home patient record. Our refinement, as described in Section VI, is also a subset of these results.

\section{B. PDA based solutions}

Already in the era of so-called Personal Digital Assistants (PDAs), there were many opportunities of handheld computing in medicine [7]. PDA's were identified as excellent tools for managing clinical information and accessing it at the point of care [8]. Some problems identified, are still relevant for modern mobile devices: e.g. a small (touch-screen) keyboard. Although several optimizations are made to mobile keyboards like using swipe gestures to enter text, alternative input methods such as Swype ${ }^{1}$ and SwiftKey $^{2}$ offer substantial benefits to users and typing speeds are comparable with common typing speeds found on computer keyboards [9]. Already in 2001, thousands of American GPs were writing prescriptions electronically and transmitting them to the pharmacy [10]. Yet, Belgian medical law requires safety precautions, like encryptions and authentication of such kind that were not possible to implement on a PDA. Nowadays the PDA is replaced by smartphones and tablets, which are often more powerful and versatile and can thus meet these requirements [11]. Our work therefore focuses on the possibility of augmenting a house visit with a mobile device in Belgium as well. The benefits of such a mobile EMR are according to Chen et al. [12] improved legibility, solving poor hand-writing and even incomplete information and more importantly, a clearly documented medication list that is more comprehensive than its paper counterpart. Grossman et al. [13] present many benefits an EMR can provide to counter adverse drug events. E.g. a renewal of medication can be prescribed more securely, since the lab results are better accessible.

\section{Usability issues in medical software}

As in most domains, usability is also a key attention point in medical applications. Schumacher et al. [14] found the following five issues: 1) inefficient workflows that do not match clinical processes, 2) confusing error messages that can be ignored amid the other demands of clinical care, 3) poorly designed screens with excessive data, 4) alert fatigue (both visual and audio) where pop-ups show up too easily [13] which results in GPs overriding them [15] and 5) frustration with what is perceived as excessive clicks during common tasks. It is important to counter these issues from the initial design. This is why we aim to: 1) determine a common workflow and design according to this workflow, 2) eliminate error

\footnotetext{
${ }^{1}$ http://www.swype.com/

${ }^{2}$ http://www.swiftkey.net/
}

messages, the design is kept simple without functionality that is not needed on a house visit, thus all possible states are covered, 3) design screens in a minimalistic manner: only the most important information - as determined during the interviews - is displayed, 4) design a dashboard where the GP can easily spot the attention points himself and 5) create the program according to a common workflow, favoring the most common actions as stressed by Williams et al [16]. We also aim that the time spent with a mobile EMR is less than with a paper equivalent. This is currently not always true. For example, prescribing medication electronically can take significantly longer ( $>25$ seconds per prescription-event) [17]. Yet, this does not hold for renewals of medication, which according to our interviews - happens a lot during a house visit. Current systems, however, do not make use of the mobile affordances offered by a mobile device. We try to make use of these affordances as much as possible to speed up the process (see Section VI). Belgian authorities complicate ambulatory prescriptions, since every prescription still has to exist on paper and GPs do not want to drag a mobile printer to every house visit. As Han et al. [18] states, social and legal issues also have to be considered when implementing an information system that might transform the shape of the health-care sector. Nevertheless, this transformation is needed. We, therefore, already include a design for a mobile prescription module for when the law is modernized - as is indeed planned.

\section{Participatory design applied in health research}

Participatory design is used before in health research. Tang et al. [19] present five design implications for applications for preterm infant care, which they incorporated into the design of Estrellita, a mobile health informatics tool to support caregivers of preterm infants. Hwang et al. [20] present a participatory design approach and the tensions that challenge user interface design for an intelligent system that assists with activities of daily living in the home. Miller et al. [21] discuss design implications in four key areas and then used their findings to suggest design strategies for youth-focused pervasive social health games. The main lesson they have learned fits our methodology best: "the only honest way to design for is to design with".

\section{E. Context sensing to improve availability}

To increase usability we want to enhance the way a GP can register observations. One way of doing this, is to provide the GP with a faster text input system. Stocky et al. [22] evaluate four ways of word suggestion: 1) language frequency, 2) user frequency, 3) recent use and 4) their own 'commonsense'. Where the commonsense approach excels in cases of low word repetition, it is not very useful for suggesting single words. Likewise, language frequency is not the best solution when entering specific medical data, as GPs often use a special jargon, this can however become interesting if we limit the language to the medical dictionary. User frequency and recent use will be used since both methods score an accuracy of more than $50 \%$. Context awareness principles (e.g. making use of the current location) help to improve the usability and usefulness. The primary context types are location, identity, time and activity [23]. The combination of these types is used to open the appropriate patient file and propose better suggestion when 
the GP enters text, resulting in a better health application. The contextual information can be obtained in a number of ways, e.g. explicitly, implicitly and inferring [24].

\section{OUR CONTRIBUTIONS}

We designed and evaluated the user interface of a mobile EMR application to be used on a house visit. We refine results from similar research [2], [3] and design a dashboard fit for mobile devices. Five core considerations that Belgian GPs are facing using a EMR during a house visit are identified and translated into five requirements: 1) exploiting mobile affordances, 2) immediate availability, 3) quick overview of most relevant data, 4) easy entry of new patient data and 5) the importance of e-prescribing. Instead of porting an existing EMR, we iteratively designed the user interface for a mobile application that certainly meets these requirements. Thus, by taking advantage of the affordances of a mobile device, including context sensing, we optimized the way a GP can use a mobile EMR application.

Nineteen GPs were interviewed during the design phase, this design is evaluated with six usability experts and the final conclusion was made based on the interviews with 53 final year general practice students, which already have done several house visits in their internships.

Furthermore, we discovered a noticeable difference between the different generations. Graduated GPs all learned to work with the SOAP note registration format. SOAP stands for Subjective, Objective, Assessment and Planning. Current students, however, find this obsolete. They prefer a more evidence based technique. This is a very interesting discovery with quite some implications on our design that we will need to further explore in follow-up studies.

\section{MEthodology}

Participatory design research is typically divided into three stages [1]: 1) initial exploration of work, 2) discovery processes and 3) prototyping. Our research is therefore also divided into these stages which are discussed in Section V. In the first stage, the researchers make arrangements with the GPs and learn the ways in which the GPs currently work, such as the used technologies and workflows, work procedures, routines and other aspects of the work. The discovery process allows the researchers and GPs to clarify the GPs' goals and values. In the last stage the researchers iteratively design and evaluate the prototypes.

Unfortunately, our researchers could not join a GP during a real-world house visit because of doctor-patient confidentiality, as he was not a GP himself. We therefore slightly had to change our initial plan of observing GPs during their daily business by interviewing them in their practice. Nineteen GPs are interviewed during the initial step during at least fifteen minutes. The GPs were asked about their opinion on the current state of the prototype. We diversified the GPs over different cities, group practices and tool-set use. Since GPs working in a group practice typically follow the same workflow, no two GPs from the same group practice were interviewed.

When following a rapid prototyping methodology, the prototype should be updated after a few users mention an issue. Nielsen [25] states that: "five users are often enough to find $85 \%$ of the usability problems, except in the case were five is not enough". Which means that when every user points out different problems, you have to test with more users. To move forward as quickly as possible, we, however, already updated the prototype when three subsequent GPs made the same remark. All prototypes are thus evaluated with small groups of minimum three physicians. Fixes are immediately applied in resemblance of the RITE (rapid iterative testing and evaluation) methodology [26]. The complete design process is described in Section VI.

The result is evaluated during a final evaluation where the test users were asked to fill in a computer aversion, attitudes en familiarity index (CAAFI) questionnaire to assess their computer skills. The CAAFI questionnaire is a seven-pointed bipolar scale, scores range from -3 to 3 . A negative score means that the user has a lower attitude and familiarity and a higher aversion. Since CAAFI is a relative larger questionnaire, this was only possible in the final evaluation due to stricter time constraints of the GPs. The usability quantified with a common questionnaire in usability research: the System Usability Scale (SUS) [27]. The complete evaluation is described in Section VII.

\section{PARTICIPATORY DESIGN PROCESS}

\section{A. Stage 1: initial exploration}

In the first stage we familiarized ourselves with existing EMR applications. Therefore, we received a demonstration of some of the most used medical record applications in Belgium: Medidoc, CareConnect, Accrimed, Medigest, MedSoft and SoSoeMe ${ }^{3}$. Since Medidoc is the most used and popular EMR in Belgium and CareConnect its successor, both EMRs are also installed on the researchers' computers to analyze. Since Belgian GPs do not consult their patient records during a house visit as there is no local mobile EMR application available on the market, we looked at popular international mobile applications like: EMR Unwired ${ }^{4}$, drChrono $^{5}$, Allscripts Wand ${ }^{6}$, Aria $^{7}$ and MediTouch ${ }^{8}$. These applications were found by searching the mobile stores for the most popular medical applications. Keywords used were: electronic medical record (EMR), electronic health record (EHR), health information system (HIS) and eHealth. All of these applications display a dashboard with patient information. From ours and the research described above appears that GPs do not need all the displayed information in these applications at first sight. Furthermore, none of these applications are especially designed to be used solely during a house visit.

\section{B. Stage 2: discovery process}

In the second phase, during the discovery process, the goal is to make meaning of the work rather than to simply

\footnotetext{
${ }^{3}$ http://www.corilus.be/documents/segments/general-practitioners/ software.xml

${ }^{4}$ http://www.sap.com/pc/tech/mobile/software/industry-apps/ patient-record-app/index.html

${ }^{5}$ https://www.drchrono.com/

${ }^{6} \mathrm{http}: / / \mathrm{www}$. allscripts.com/wand

${ }^{7}$ http://www.varian.com/us/oncology/radiation_oncology/aria/

${ }^{8}$ http://www.healthfusion.com/
} 
describe it. A questionnaire ${ }^{9}$ is used to get first initial insights about the workflow of GPs during a typical house visit. We used this questionnaire to guide the initial talks with several stakeholders: two GPs, a usability expert, a human computer interaction professor and the head designer of an EMR system. The outcome of these first talks resulted, in our initial sketches that are used in the first prototyping iteration.

\section{Stage 3: prototyping}

The prototyping stage is divided into three steps with different target audiences. The prototype is iteratively designed with nineteen GPs. Afterwards the prototype was improved with the help of six usability experts. The final evaluation is performed with 53 final year students (see also Figure 8). All interviews can reveal possible mistakes or missing features that can be added in a next prototype.

1) Step 1 - GPs: With the GPs, we iteratively sought feedback on the designs. Most interviews involved one GP and one researcher, except for two interviews with one researchers and two GPs. As pictures say more than a 1000 words to explain ideas, low fidelity paper prototypes were initially used during the interviews to gather early feedback in the design process. To make it easier for the test user to see the prototype as an actual tablet application, the prototypes are surrounded with the same borders as a tablet [28]. The last prototype, however, is already a real-life android application on a tablet. The researcher carefully noted down all major issues with the particular prototypes to solve them in following iterations. The GPs were asked to elaborate on the whole workflow. Questions asked were: who answers the phone? Who configures the GPS? What kind of information or material do you carry with you during house visits? Do you already use an EMR application or, if not, what kinds of patient information do you take with you? Which data is entered in the EMR application (after the house visits)? How do you prescribe medicine? Do you take pictures of wounds or rash? What kind of patients do you normally treat during a house visit?

2) Step 2 - usability experts: To eliminate possible usability errors in our design, six usability experts were interviewed. They were interviewed in sessions of one hour. We presented a typical scenario - which we learned from the GPs - to these experts. It did serve as a validation for the design that was put forward during the third phase. With this iteration, it was possible to improve some mistakes, so the real target audience (whom are hard to schedule interviews with) will not lose time on these issues in the next evaluation.

3) Step 3 - general practice students: The final evaluation was performed in three sessions of 30 minutes with in total 53 general practice students in their final year, with approximately 120 hours experience in general practice. Each session started with a presentation of the current iteration, followed-up with an online questionnaire. As in a participatory design approach, it is important to know the users, the students were asked to fill in the CAAFI questionnaire. Thereafter they were asked if they experienced the same problems during their internships as graduated GPs and finally each screen of the final design was shown. Each screen was graded using a five point Likert

\footnotetext{
${ }^{9}$ http://goo.gl/Wxh3Gi
}

scale [29] and discussed in an open class discussion. The total score was measured with a SUS questionnaire [27].

\section{DESIGN DESCRIPTION}

This section elaborates on the first step of Stage 3. The design of the prototype through rapid prototyping with the GPs. We used the first five out of nineteen interviews to build an overview on the various steps during a typical house visit. This was then translated into design requirement for the mobile EMR application. The same questions were asked to all five GPs to make sure a common answer could be extracted. Finally, five main considerations could be revealed, which were then confirmed by the other fourteen GPs.

1) not optimized for mobile: the screen of a mobile device is not big enough to display the desktop interface of the EMR application (Figure 1). Even a 13 inch screen is too small to fully use the current EMRs.

2) too slow: GPs can access their EMR application through a terminal server connection on a laptop, often a low budget laptop that boots slowly. Due to a long login process, it takes a long time before the laptop is connected to the internet. Furthermore, it is often the case that the battery's lifetime is too short for a complete tour of patients. Finding a power socket with patients means losing precious time and is not always possible. Only one GP had an ultrabook, so for him this issue does not apply.

3) necessary patient information: it appeared that GPs always memorized or printed the same kind of information before going on house visits. This information is translated into the elements that are present on the dashboard of the first prototype (Table I).

4) registering data: only $10 / 19$ of the interviewed GPs register data into the EMR application after a house visit. Whenever data is entered, this is always done in a structured way: the subjective and objective observations, the assessment, the treatment and the planning. This structure is called SOAP and is a problem-oriented way to register information as discussed by Tange [30].

5) prescribing medicine: currently Belgian law obligates the use of a paper prescription, therefore GPs do not register their prescriptions in their EMR application. They do not want to do double work of writing a prescription on paper and enter it again digitally.

The design proposed in this paper aims to tackle these five considerations and thus provide GPs with a usable and useful mobile EMR application. Each issue is translated into a requirement and is discussed in its respective place. These issues are common issues and requirements that many mobile systems have that show information and allow to diagnose or enter information about the local situation, yet we offer an optimized solution for a mobile EMR. Furthermore, we actively tried to find a workflow that GPs follow during a house visit: $15 / 19$ of the GPs indicated that they first opened the most important patient information and reviewed the messages and/or lab results before entering their findings into the EMR application. 13/19 prescribe medicine as the last part of the house visit; the other $6 / 19$ did this during the last part of the 


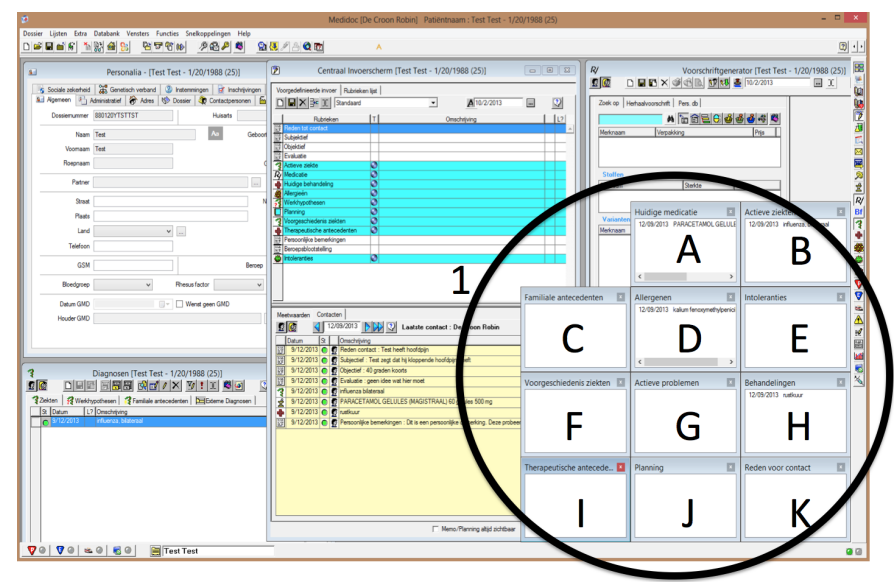

Fig. 1: Screenshot from Medidoc with the most used documents open. This interface is developed with larger, nontouch screens in mind. The 'Medidoc mosaic' (1), contains the information that is often printed by the GPs and brought along on a house visit: medication (A), active diseases (B), family history $(C)$, allergens $(D)$, intolerances $(E)$, prehistory $(\mathrm{F})$, active problems $(\mathrm{G})$, treatments $(\mathrm{H})$, therapeutic history $(\mathrm{I})$, planning $(\mathrm{J})$ and reason for contact $(\mathrm{K})$.

SOAP registration. A tablet interface is chosen since 17/19 GPs indicated that they preferred a tablet for their mobile EMR application.

\section{A. Requirement 1: mobile affordances}

Instead of porting an existing desktop program to a mobile application, we take advantage of the possibilities that a mobile device offers. Porting menus and making buttons larger is not desirable. Most mobile devices have much more affordances ${ }^{10}$ : a multi-touch screen offers novel gestures that are not available on a traditional computer, a GPS sensor allows to immediately open the right patient record based on location, a better battery lifetime lasts for a whole day of house visits without the need of charging, the low weight and a small size makes it easy to bring along, speech-to-text allows for easy patient data entry, the camera allows to take pictures of e.g. wounds or rash for easy follow-up, Bluetooth or NFC allows to sync with other medical devices, etc. It is clear that these affordances can drastically augment the existing applications. Since only the desired functionality is included, the interface can be kept clean and minimal without a clutter of buttons and menus. Advanced functionality of a typical EMR that is not needed on a house visit it thus not included into the design.

\section{B. Requirement 2: show timely and relevant information}

The right patient file is opened automatically based on a combination of the GPS location in combination with the GP's calendar. If the system is incorrect or cannot determine the correct patient, it will provide the GP with good patient candidates. Doing so, the GP will not lose time searching for the correct patient file. When the correct patient file is opened, the GP sees a dashboard with the most relevant patient information (discussed in Requirement 3). The GP can

\footnotetext{
${ }^{10}$ not necessarily limited to a mobile device
}

TABLE I: The number indicates how many GPs ranked this dashboard element as important. The prototypes are tested with five, three, three, three and five user respectively. The gray rows are the elements displayed on the final dashboard.

\begin{tabular}{|l|c|c|c|c|c|}
\hline \multirow{2}{*}{ Element on dashboard } & \multicolumn{5}{|c|}{ Prototype } \\
\cline { 2 - 6 } & $\mathbf{1}$ & $\mathbf{2}$ & $\mathbf{3}$ & $\mathbf{4}$ & $\mathbf{5}$ \\
\hline active medication & $5 / 5$ & $3 / 3$ & $3 / 3$ & $3 / 3$ & $5 / 5$ \\
\hline active problems & $5 / 5$ & $3 / 3$ & $3 / 3$ & $3 / 3$ & $5 / 5$ \\
\hline active diseases & $4 / 5$ & $2 / 3$ & $1 / 3$ & N/A & N/A \\
\hline intolerances & $4 / 5$ & $3 / 3$ & $3 / 3$ & $3 / 3$ & $5 / 5$ \\
\hline allergens & $5 / 5$ & $3 / 3$ & $3 / 3$ & $3 / 3$ & $5 / 5$ \\
\hline history of diseases & $5 / 5$ & $2 / 3$ & $3 / 3$ & $3 / 3$ & $5 / 5$ \\
\hline family history & $3 / 5$ & N/A & N/A & N/A & N/A \\
\hline therapeutic antecedents & $2 / 5$ & N/A & $1 / 3$ & N/A & N/A \\
\hline treatments & $3 / 5$ & N/A & $1 / 3$ & N/A & N/A \\
\hline free notes & N/A & $0 / 3$ & N/A & N/A & N/A \\
\hline last lab results & N/A & $3 / 3$ & $3 / 3$ & $3 / 3$ & $5 / 5$ \\
\hline picture & N/A & $0 / 3$ & N/A & N/A & N/A \\
\hline
\end{tabular}

furthermore easily search patient related documents such as: lab results, referral letters and radiographic images. Finally, the GP can register the patient contact using the SOAP structure. Only displaying the most important data on the dashboard, saves time because the GP does not have to navigate through irrelevant information. The right screen will appear on the right time.

\section{Requirement 3: a quick overview of the most important patient data}

The first screen GPs see is a dashboard with the most important data about the current patient they are visiting. The content of this dashboard was adapted three times (see also Figure 8). The first dashboard is shown in Figure 2. With the help of the five different prototypes, we were able to refine the results from Abd Ghani [2] and Plancke [3] to a smaller subset. Table I shows the evolution of the dashboard over the different prototypes and indicates how we started with a larger set and refined this set with the help of the next three iterations. The final dashboard is shown in Figure 3.

With each interview the GPs were asked if all needed information was present. To obtain a priority between the elements, they also had to choose the three most important elements and three elements that could be deleted. Active medication, problems and allergens are constantly ranked important by all nineteen GPs. 18/19 GPs rate intolerances and history of diseases important. Active diseases were only rated important in the first prototype (4/5), while in the following prototypes, it became clear that active diseases are better listed as active problems, hence the decline in importance: $2 / 3$ and $1 / 3$ in the second and third prototype respectively. On the other hand, when asked what could be deleted if necessary, the GPs unanimous indicated: family history, therapeutic antecedents and treatments. We used the place of these elements to introduce some other elements on the dashboard of the second prototype: free notes, last lab results and a medical picture of that patient taken by the GP. The last lab results were very well received (14/14). Especially during a house visit, where chronic cases are most common, it happens a lot that the GP needs the last lab results. Free notes and a picture appeared to be superfluous as $0 / 3$ GPs indicated that they will use it. Since we want to reduce the elements on the dashboard, we reintroduced 


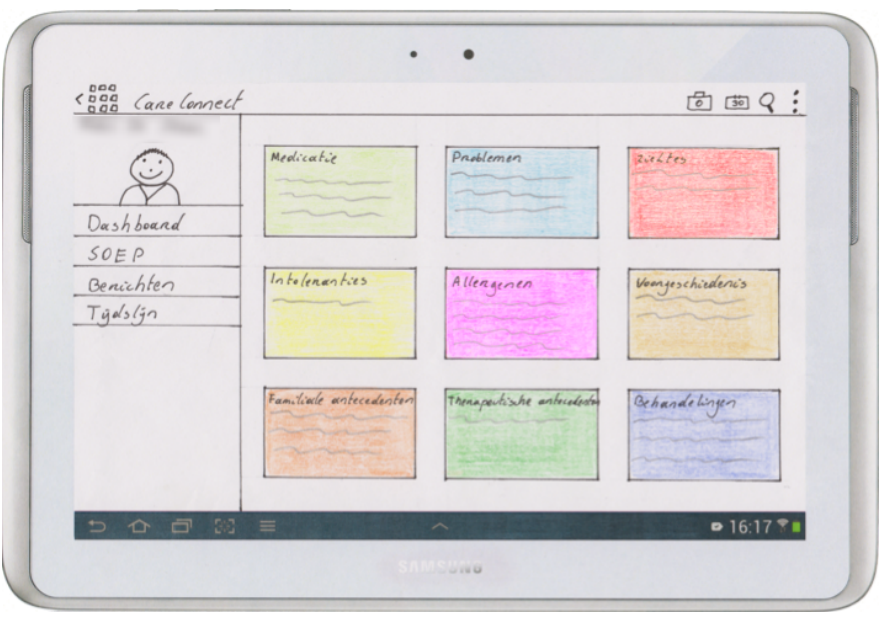

Fig. 2: The first paper prototype. Every element is colored with a different color for illustration purposes: medication (light green), problems (light blue), diseases (red), intolerances (yellow), allergens (dark pink), history of diseases (orange), family history (brown), therapeutic antecedents (dark green) and treatments (dark blue).

treatments and therapeutic antecedents for confirmation that GPs rank these two as the least important.

Thanks to the different prototypes we could reduce the number of elements on the fourth version of the dashboard from nine to six. This enhances the visibility of these remaining elements. The fifth prototype was used to research which additional information is needed for the finally selected elements on the dashboard. For example, for each active medication show the start date and dosage. We did, however, not yet look into the motivations behind our results. E.g. allergens could be ranked important because the GPs are not aware that pharmacovigilance ${ }^{11}$ is applied. We will try to find the motivation of the remaining elements on the dashboard in a next prototype. The removed elements are currently not accessible, if in further research appears that the GPs still want to access these elements, the dashboard could be made scrollable.

\section{Requirement 4: an efficient and effective way to register patient data}

Since the job of a GP is to practice health care and not to enter data, registering information should be fast and easy. We therefore also iterated over the five different prototypes to improve the way GPs could register patient data according to the SOAP structure. The first two prototypes used the traditional input forms, augmented with speech-to-text and digital handwriting recognition. After the second prototype, eight GPs were interviewed already. Hence, we had a clearer view of the needs of the GPs and the data registration screens were enhanced with a text suggestion system as described by Stocky et al. [22]. We are currently negotiating to get access to some medical databases to solve the cold start problem, where not enough data is present for input recommendations [31]. Four different kind of input screens are used during the SOAP

\footnotetext{
${ }^{11}$ detection of possible medication aversion effects
}

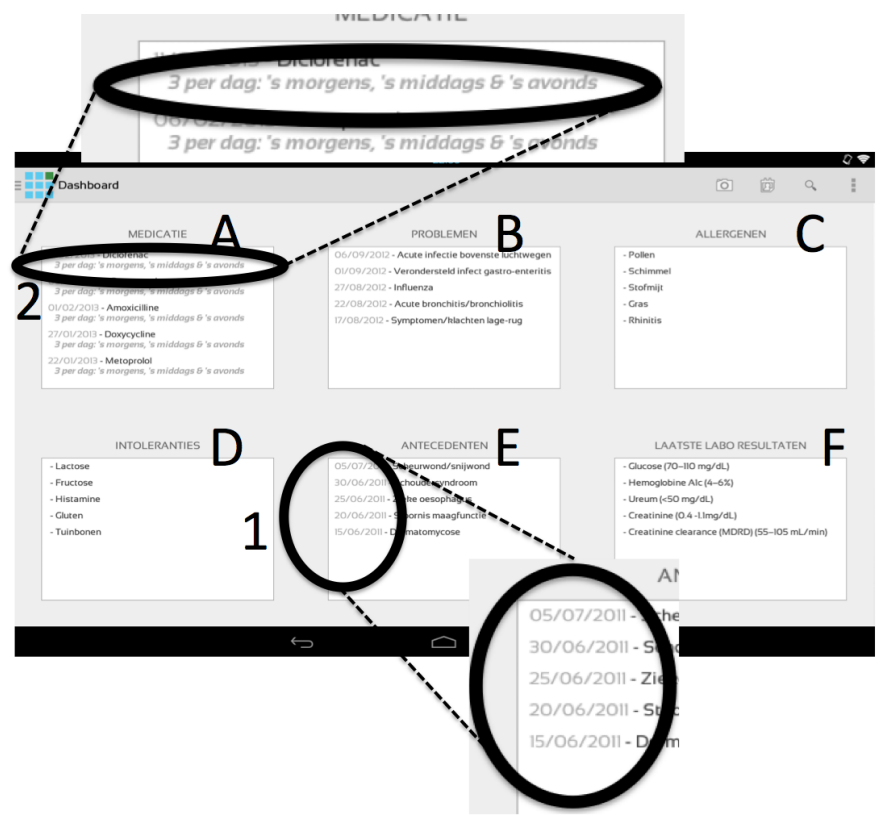

Fig. 3: The sixth final elements on the dashboard of the last prototype: medication (A), problems (B), allergens (C), intolerances (D), history (E), lab results (F). The additional information is enlarged for readability: the dosage for medication (1) and the date (2).

registration: the subjective screen is used to enter the comments the patients tells the GP, under the objective the observations of the GP himself are registered, the assessment screen enables the GP to enter a certain disease and the planning screen is used to register a roadmap that should be followed to cure the patient.

- The subjective screen (Figure 4) proposes suggestions based on favorites, most used, recent use, patient history, colleagues and location. Most used and recent use are integrated because of the high accuracy [22]. Since all GPs indicated that chronic cases are the most common on a house visit, the data is often the same for that patient. Hence, we can suggest words, based on the patient's history. Colleagues working in the same group practice often encounter similar problems; a suggestion system based on what their colleagues enter is thus valuable and integrated in our design. We finally include a location based suggestion system to provide easy input for contagious cases such as the flu. Favorites can be set to speed up other combinations the GP likes to use. The GP can either select a word or use drag-and-drop to use the suggested words. In case the GP can not find the correct word, the input methods from the second prototype can be used.

- The objective screen combines a text input screen like the subjective screen with sliders and incremental input fields to enter parameters such as temperature or blood pressure. The data fields are not empty but already contain clearly indicated previously measured values, so optimally the GP would not need to change this, but can change it incrementally. The sliders can 


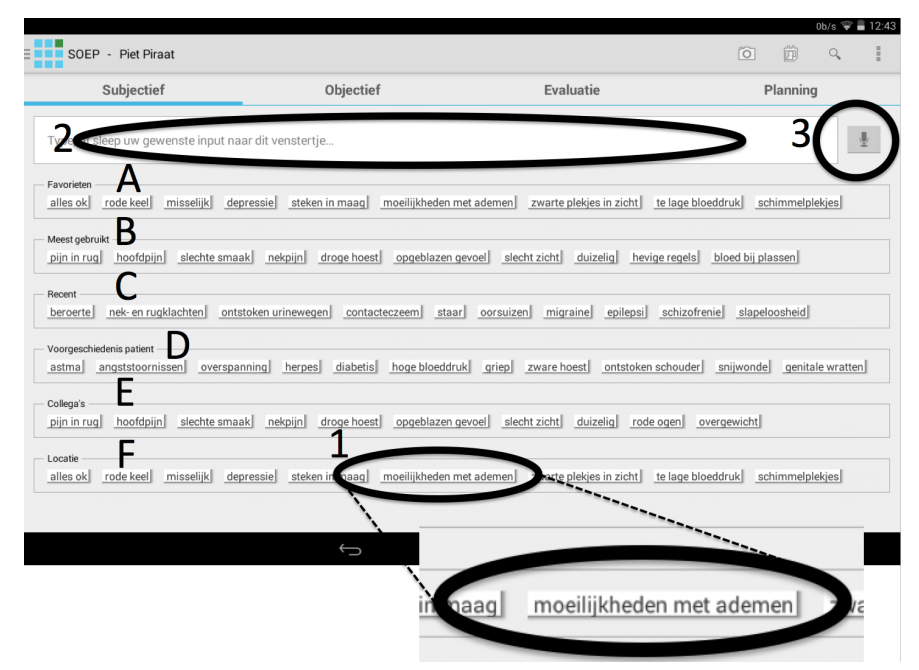

Fig. 4: Subjective input screen, note the different categories where the user can select words (1): favorites (A), most used (B), recent use (C), history of the patient (D), colleagues (E) and location $(\mathrm{F})$. He can also freely type (2) or use text-tospeech (3)

also be used to indicate the normal 'healthy' ranges. GPs however remarked that they already know the normal ranges, but as Weibel et al. [6] indicate, a EMR application can also be used to communicate health information to the patient. Most measures could be registered automatically: the heart rate can be measured directly with the camera of the mobile device (even ECG measurements are possible with FDA approved ${ }^{12}$ sensors $^{13}$ ), the blood pressure could be registered automatically with a Bluetooth enabled blood pressure monitor, like for example the Withings' Blood Pressure Monitor ${ }^{14}$. All kinds of trackers can be added.

- In the assessment screen the GP can select an element from the $\mathrm{ICPC}^{15}$ coding. The different chapters of ICPC completely fill the screen, which makes it easy for the GP to select an element with a touch gesture, in case free text needs to be entered, an automatic suggestion text box is still provided. As soon as the user starts typing, suggestions are presented.

- The planning screen displays options to register a possible future follow up. For reason of consistency, it is built similarly as the subjective screen with several word suggestions: favorites, most used, recently used, treatments, referrals and individual parameters.

\section{E. Requirement 5: incentives to prescribe medication digitally}

A lot of important information is lost if GPs do not register their medicine prescriptions into the electronic patient file. Yet the Belgian law obligates the use of a paper prescription. While

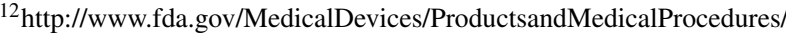
DeviceApprovalsandClearances/510kClearances/ucm330634.htm

${ }^{13} \mathrm{http}: / / \mathrm{www}$.alivecor.com/

${ }^{14} \mathrm{http} / / / \mathrm{www}$.withings.com/bloodpressuremonitor

${ }^{15}$ http://www.who.int/classifications/icd/adaptations/icpc2/
}

the law is not modernized, the GPs need to be motivated to not only write the prescription, but also digitally enter it into their EMR. As nobody wants to do redundant work, neither do GPs. Multiple solutions were considered and discussed during the interviews: mobile printers are considered to be too heavy, digital pens (e.g. Livescribe ${ }^{16}$ ) too expensive and taking a picture with the integrated camera too cumbersome. Furthermore, it is more usable if the GP can prescribe drugs without additional tools. Regardless of the law, a fast input method is necessary. An incentive, like pharmacovigilance, can convince GPs to do this redundant work. To make it as easy as possible, the same input methods are used as with Requirement 4. Medication suggestions are based on key words in the SOAP, most and recently used, chronic medication, medication that the patient has taken before, location and family basis. Currently there are two projects under development in Belgium to send a digital prescription to the pharmacist: vitalink ${ }^{17}$ and recip- $\mathrm{e}^{18}$. A bar code scanner is integrated to scan existing medication packaging. According to GPs, it happens that a patient approaches a GP with a medication package and just asks for a renewal of this particular medication. Thanks to the scanner, the medication can be loaded into the prescription module.

\section{USABILITY EVALUATION}

After the initial design phase with the GPs, usability experts were asked to evaluate the current design. We thereafter evaluated the prototype with 53 final year general practice students, but in order for the students to focus on the important matters, the experts were asked their opinion first. This way, no time is lost on problems non-GPs can also detect.

\section{A. Usability experts}

In total, six experts were interviewed in one-hour sessions. They first received an explanation of the context and a variety of typical scenarios of a house visit of a GP. They are aware of the methodology and are familiar with the working method.

1) Remarks: 6/6 indicate that it should be possible to navigate from the dashboard immediately to all lab results, which makes sense and thus this function will be added. Other individual remarks were: add a filter and search option. When in the office, show the calendar so the GP has a clear overview of all house visits. Let the GP change the profile picture of the patient immediately with the camera. Also show the visits of the GPs colleagues in the consultation overview, this is similar to remarks made by two GPs and can be included into the next prototype.

2) SUS score: Although not entirely representative, the SUS score of the experts provides an indication about the general usability. With an average SUS score of 80, the usability of the application can be listed as 'good' [27].

\section{B. Students}

Fifty-three final year general practice students were questioned to evaluate the latest version of the prototype. They

\footnotetext{
${ }^{16} \mathrm{http}: / / \mathrm{www}$. livescribe.com/

${ }^{17} \mathrm{http}: / /$ www.vitalink.be/

${ }^{18}$ http://recip-e.be/
} 


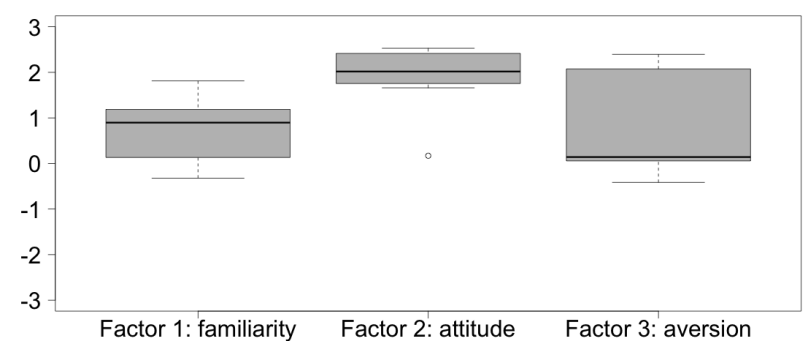

Fig. 5: The distribution of the CAAFI scores of the students. The positive attitude towards computers is clearly visible.

were evaluated during an advanced EMR class. The complete questionnaire was divided into three parts: 1) the CAAFI questionnaire [32], 2) a screen design evaluation and 3) a SUS questionnaire. A general feedback input field was also included, combined with a class discussion. Three students however forgot to click the submit button of the second and third questionnaire. Therefore we have a population of $n=53$ for CAAFI and $n=50$ for the other questions. The gender was equally distributed with 27 males and 26 females, the average age $25.32(\mathrm{sd}=4.26), 77 \%$ have a smartphone, $32 \%$ have a tablet, $15 \%$ have neither and $25 \%$ have both.

1) CAAFI questionnaire: The resulting scores of the CAAFI questionnaire are displayed in Figure 5. The average score of $0.76(\mathrm{SD}=0.75)$ on Factor 1 indicates that the students are slightly familiar with computers and have a rather positive attitude towards computers according to the average value of $1.91(\mathrm{SD}=0.69)$ on Factor 2. The average aversion score on Factor 3 was $0.68(\mathrm{SD}=1.1)$ (a positive number means low aversion) and indicates the students have some aversion towards computers.

2) Screen design evaluation: The questions were asked as a five-level Likert item ranging from very poor to very good. Figure 6 shows the distributions of the answers (very good + good). $96 \%$ of the students $(26 \%+70 \%)$ think the dashboard is well designed. The patient information screen scores positive with $83 \%(36 \%+50 \%)$ and the document screen scores $82 \%$ $(14 \%+68 \%)$. The SOAP screens scores are more divided: Only $40 \%(4 \%+36 \%)$ thought positive of the subjective screen, the objective screen scored a little higher with $52 \%(8 \%$ $+44 \%)$ positive scores. The evaluation screen managed to get $68 \%(8 \%+60 \%)$ positive scores; the planning screen scored worst with only $22 \%(3 \%+19 \%)$ positive scores. During the class discussion all students reported that they would prefer a more evidence based registration system compared to the SOAP system. This means that the design should be less text input oriented and more focused on visualizing the data from evidence based databases.

3) SUS questionnaire: The students gave an average score of 70. The distribution of the different scores are displayed in Figure 7 and discussed in Section VIII.

\section{DISCUSSION}

In Belgium there are strong regulations for developing a medical application. As a result, only nine EMRs are allowed

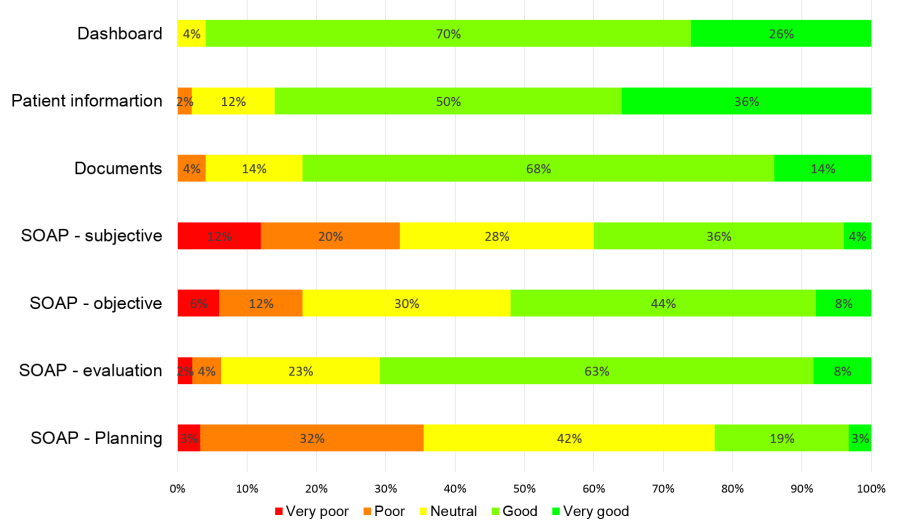

Fig. 6: Distribution of the scores on every screen. Dark green is very good, green is good, yellow is neutral, orange is poor and red is very poor.

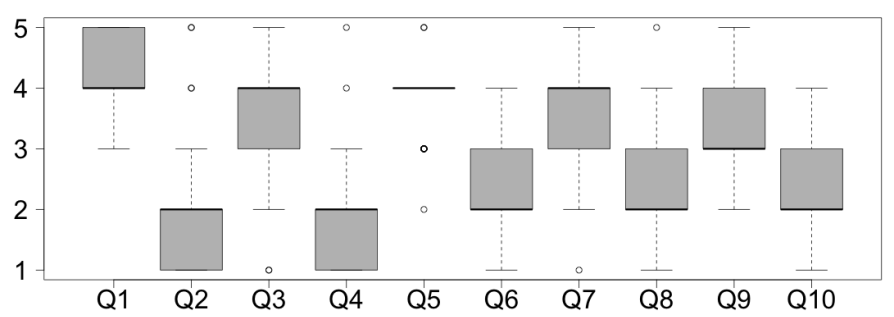

Fig. 7: The distribution of the SUS scores from the students on the different questions. A high score means the user completely agrees with the statement. The SUS questions are ordered to create alternating scores in the ideal situation.

on the market ${ }^{19}$. The most used product is Medidoc, which exists for more than 25 years and evolved gradually to its current version [33]. During the interviews, we noticed that the use of a particular EMR strongly determines the workflow of the GP. We discovered that GP needs are dependent on the EMR program they use, while it should be the other way around: the tools should be adapted to the needs of the GPs. Medidoc is a typical multi documents interface ${ }^{20}$ and the user can configure the layout to his or her needs. This makes it more difficult to discover a common workflow. Yet we managed to extract a common workflow that most GPs follow during a house visit as described in Section VI. Since time is of the essence, we optimized the way a GP can use a mobile EMR application. The right patient file is automatically opened, wherein the needed patient data is immediately displayed on the dashboard. GPs can enter their findings in an effective and efficient way thanks to context enhanced registration process. However, additional tests are needed to prove actual time improvements and show the actual usability and fitness for purpose in real life situations.

The different prototypes allowed us to make concrete examples of the concepts for early visualization, verification, iteration, and optimization. Our design was developed following this principle and we used the early development of

\footnotetext{
${ }^{19}$ https://www.ehealth.fgov.be/nl/registratie-van-de-medische-softwarepakketten

${ }^{20} \mathrm{http}: / / \mathrm{msdn} . \mathrm{microsoft} . c 0 m /$ en-us/library/windows/desktop/ms632591
} 


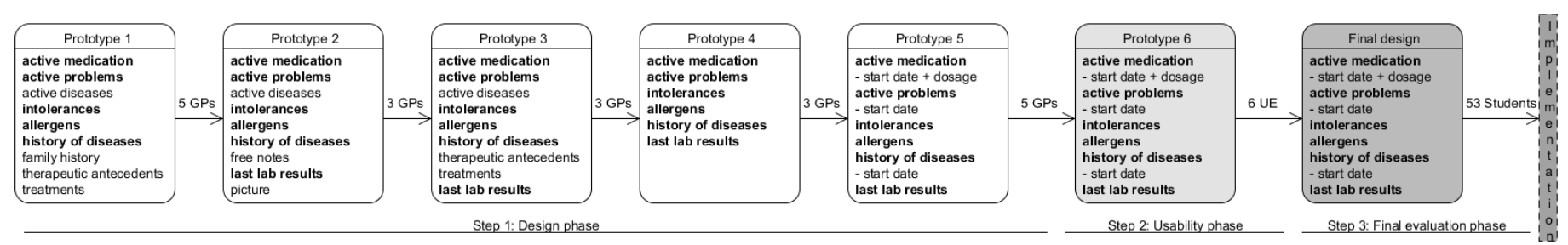

Fig. 8: Timeline that shows the prototypes. In the first step five prototypes (white) were evaluated with GPs, six usability experts were questioned in the second step (light gray), finally 53 students evaluated the final design (dark grey) in the third step.

the design to conduct some evaluation sessions. After almost every interview we received valuable feedback that could be used to make minor changes to the design. Whenever at least three GPs mentioned a specific flaw, we tried to find a solution that we could show to the next GPs. When a comment about this solution arose in the next interview, we also showed the previous version and tried to get insight about what would work better, or if we needed to come up with a third solution. In this way, we could move forward relatively quickly. It appears that the relatively short time provided for each interview did not have a huge impact. Changes made to the prototype were most of the time positively received. Thanks to this approach and a thorough preparation of each interview, we were able to work with several prototypes (that could be divided in five major versions). The fifth prototype was tested again with five users to be more certain about the results.

The scores from the evaluation with the final year general practice students were positive. Screens without SOAP elements were well received. This strengthens our findings that the six elements on the dashboard are well chosen. By using the participatory design approach, we found a major discrepancy between the different generations. The students do not use SOAP anymore, but are taught a more evidence based methodology. They are used to the system of a medical history (anamnesis), clinical examination, diagnosis and a treatment plan. This produces several improvements for a mobile EMR system. Text that needs to be registered can be shorter and better suggestion systems, supported by medical evidence can be implemented. It is rather remarkable that none of the GPs we spoke, mentioned this newer system. They are used to their SOAP system and prefer to work this way. It is important to know this difference when designing a new EMR. This can also explain the lower scores of the SOAP screens (Figure 6). When asked afterwards, the students were positive over the text suggestions, yet they just not liked the amount of text that is needed for SOAP.

During the first step of phase 3 , we did not perform a SUS questionnaire as usual, because of time constraints of the short interview. We realize that we potentially missed useful quantitative feedback in this early phase of design. We did, however, ask the six experts and fifty students (three students forgot to submit) to fill in the questionnaire to get a general view on how our design compares to other applications. The experts gave an average score of 80 , which according to Bangor [27], list the application as 'good'. The ninth and tenth question: "I would imagine that most people would learn to use this system very quickly" and "I needed to learn a lot of things before I could get going with this system." are responsible for pulling down the score. This could be explained by the fact that the experts have no medical background and could not understand all the terms. The total score of the students is, however, 70. A possible indication of the lower score could be that these students learned to work with a newer registration system and think SOAP is obsolete. This is an important discovery for all that want to design medical applications in Belgium (see also Figure 6, where all nonSOAP screens are positively graded). An important remark is that the ninth question ("I felt very confident using the system") scored lowest again. This time, it could be explained with the CAAFI results (Figure 5), and by an input system they are not used to. But since it also scored low with the experts, more research is needed to explain this finding.

\section{FUtURE WORK AND CONCLUSION}

This paper presented a case study on finding user requirements for a mobile EMR system through a participatory design approach. We have analyzed and presented the most important patient information for a GP during a house visit. We have furthermore refined the data sets proposed in earlier research [2], [3] to a smaller subset: active medication, active problems, allergens, intolerances, history of diseases and latest lab results.

We have identified five core considerations related to the use of an EMR application during a house visit and translated these considerations into five design requirements. These were the importance of exploiting mobile affordances (like touch and sensor based input), the immediate availability of relevant information, the provision of a quick overview of the most relevant data, enabling easy entry of new patient data and the importance of incentives for digital prescriptions. Our approach shows how one can use a participatory design approach to carry out a requirement analysis while iterating over subsequent versions of the user interface at the same time. For example, we discovered a major discrepancy between different generations of GPs. The older generation uses and wants to keep using the SOAP system, while the new generations is taught a more evidence based system. It is thus worth further research to offer a flexible solution that can address both paradigms, since both generations of GPs would need assistance.

We designed the user interface for a mobile application that is currently under actual development as a cross-platform application of which several GPs already pledged to use. A follow-up study of these GPs can prove if the design also works in real practice, whether the automatic patient selection based 
on GPS and calendar data works sufficiently well and whether there are features missing in the final design. Future work will focus on the difference between SOAP and the more evidence based system and should confirm if our findings are applicable outside the Belgian market, where graduated physicians are not necessarily used to work with the SOAP note system and medical law could impose different requirements. Finally, as a GP can inform a patient about his health status more easily with a mobile EMD [6], it is interesting to research this possible opportunity.

\section{ACKNOWLEDGMENT}

This research was funded under research grand IWT 120896. The authors would like to thank the participating GPs and students for their feedback and time in their already busy schedule. The Academic Centre for General Practice of Leuven and Corilus for access to their programs. We also want to thank the usability experts for their extensive feedback.

\section{REFERENCES}

[1] C. Spinuzzi, "The methodology of participatory design," Technical Communication, vol. 52, no. August 2004, pp. 163-174, 2005.

[2] M. K. Abd Ghani, "The design of flexible Pervasive Electronic Health Record (PEHR)," in 2011 IEEE Colloquium on Humanities, Science and Engineering, no. Chuser. IEEE, Dec. 2011, pp. 249-254.

[3] L. Plancke, "Het thuisdossier voor de wachtarts : stand van zaken," Master's thesis, Universiteit Gent, 2013. [Online]. Available: http://lib.ugent.be/fulltxt/RUG01/002/002/163/ RUG01-002002163 \_2013\_0001 \_AC.pdf

[4] P. Barach and S. D. Small, "Reporting and preventing medical mishaps: lessons from non-medical near miss reporting systems." BMJ (Clinical research ed.), vol. 320, no. 7237, pp. 759-63, Mar. 2000.

[5] J. Smith, "Building a safer NHS for patients: Improving Medication Safety," Department of Health, London, Tech. Rep., 2004.

[6] N. Weibel, C. Emmenegger, J. Lyons, R. Dixit, L. Hill, and J. Hollan, "Interpreter-Mediated Physician-Patient Communication: Opportunities for Multimodal Healthcare Interfaces," in Proceedings of the ICTs for improving Patients Rehabilitation Research Techniques. IEEE, 2013.

[7] S. Fischer, "Handheld Computing in Medicine," Journal of the American Medical Informatics Association, vol. 10, no. 2, pp. 139-149, Nov. 2002

[8] M. H. Ebell, D. L. Gaspar, and S. Khurana, "Family physicians' preferences for computerized decision-support hardware and software." The Journal of family practice, vol. 45, no. 2, pp. 137-141, 1997.

[9] T. Page, "Usability of text input interfaces in smartphones," J. of Design Research, vol. 11, no. 1, p. 39, 2013.

[10] J. Kelly, "Going wireless." Hospitals \& health networks / AHA, vol. 74, no. 11 , pp. $65-66,68,2000$.

[11] M. Nyssen, K. Thomeer, and R. Buyl, "Generating and transmitting ambulatory electronic medical prescriptions," in XII Mediterranean Conference on Medical and Biological Engineering and Computing 2010, P. Bamidis and N. Pallikarakis, Eds. Springer Berlin Heidelberg, 2010, pp. 890-892.

[12] Y. Chen and M. E. Atwood, "Challenges of Mobile Clinical System Design: What do Nurses Think?" in 2010 43rd Hawaii International Conference on System Sciences. IEEE, 2010, pp. 1-9.

[13] J. M. Grossman, A. Gerland, M. C. Reed, and C. Fahlman, "Physicians' experiences using commercial e-prescribing systems." Health affairs (Project Hope), vol. 26, no. 3, pp. w393-404, 2007.

[14] R. M. Schumacher, L. Berkowitz, P. Abramson, and D. Liebovitz, "Electronic Health Records: Physician's Perspective on Usability," Proceedings of the Human Factors and Ergonomics Society Annual Meeting, vol. 54, no. 12, pp. 816-820, Sep. 2010.
[15] H. van der Sijs, J. Aarts, A. Vulto, and M. Berg, "Overriding of drug safety alerts in computerized physician order entry." Journal of the American Medical Informatics Association : JAMIA, vol. 13, no. 2, pp. 138-47, 2006

[16] W. G. Williams and J. M. Morgan, "The clinician-information interface." Medinfo. MEDINFO, vol. 8 Pt 1, pp. 801-5, Jan. 1995.

[17] E. B. Devine, W. Hollingworth, R. N. Hansen, N. M. Lawless, J. L. Wilson-Norton, D. P. Martin, D. K. Blough, and S. D. Sullivan, "Electronic prescribing at the point of care: a time-motion study in the primary care setting." Health services research, vol. 45, no. 1, pp. 152-71, Mar. 2010.

[18] S. Han, V. Harkke, P. Mustonen, M. Seppänen, and M. Kallio, "Mobilizing medical information and knowledge: some insights from a survey," in European Conference on Information Systems, 2004.

[19] K. Tang, S. Hirano, K. Cheng, and G. Hayes, "Balancing Caregiver and Clinician Needs in a Mobile Health Informatics Tool for Preterm Infants," in Proceedings of the 6th International Conference on Pervasive Computing Technologies for Healthcare. IEEE, 2012, pp. 1-8.

[20] A. Hwang, K. Truong, and A. Mihailidis, "Using participatory design to determine the needs of informal caregivers for smart home user interfaces," in Proceedings of the 6th International Conference on Pervasive Computing Technologies for Healthcare. IEEE, 2012, pp. $41-48$.

[21] A. Miller, J. Pater, and E. Mynatt, "Design Strategies for YouthFocused Pervasive Social Health Games," in Proceedings of the ICTs for improving Patients Rehabilitation Research Techniques. IEEE, 2013.

[22] T. Stocky, A. Faaborg, and H. Lieberman, "A commonsense approach to predictive text entry," in Extended abstracts of the 2004 conference on Human factors and computing systems - CHI '04. New York, New York, USA: ACM Press, 2004, p. 1163.

[23] A. K. Dey and G. D. Abowd, "Towards a Better Understanding of Context and," Computing Systems, vol. 40, no. 3, pp. 304-307, 1999.

[24] G. Adomavicius and A. Tuzhilin, Recommender Systems Handbook, F. Ricci, L. Rokach, B. Shapira, and P. B. Kantor, Eds. Boston, MA: Springer US, 2011.

[25] J. Nielsen, "Why You Only Need to Test with 5 Users." [Online]. Available: http://www.nngroup.com/articles/ why-you-only-need-to-test-with-5-users/

[26] D. Wixon, "Evaluating usability methods: why the current literature fails the practitioner," interactions, vol. 10, no. 4, p. 28, Jul. 2003.

[27] A. Bangor, P. Kortum, and J. Miller, "Determining what individual SUS scores mean: Adding an adjective rating scale," Journal of usability studies, vol. 4, no. 3, pp. 114-123, 2009.

[28] M. de Sá and L. Carriço, "Low-Fi Prototyping for Mobile Devices," in CHI '06 Extended Abstracts: Human Factors in Computing Systems. ACM, 2006, pp. 694-699.

[29] R. Likert, "A technique for the measurement of attitudes," Archives of Psychology, vol. 22 140, p. 55, 1932.

[30] H. Tange, "How to approach the structuring of the medical record? Towards a model for flexible access to free text medical data," International Journal of Bio-Medical Computing, vol. 42, no. 12, pp. 27-34, 1996.

[31] A. I. Schein, A. Popescul, L. H. Ungar, and D. M. Pennock, "Methods and metrics for cold-start recommendations," in Proceedings of the 25th annual international ACM SIGIR conference on Research and development in information retrieval - SIGIR '02. New York, New York, USA: ACM Press, 2002, p. 253.

[32] S. E. Schulenberg and A. M. Melton, "The Computer Aversion, Attitudes, and Familiarity Index (CAAFI): A validity study," Computers in Human Behavior, vol. 24, no. 6, pp. 2620-2638, Sep. 2008.

[33] J. Van Damme, Het digitaal papieren dossier overstijgen. Een persoonlijke reflectie bij 25 jaar Medidoc. Uitgeverij Acco C.V., 2013. 